\title{
The Vulnerability of Some Networks including Cycles via Domination Parameters
}

\author{
Tufan Turaci and Hüseyin Aksan \\ Department of Mathematics, Faculty of Science, Karabük University, 78050 Karabük, Turkey \\ Correspondence should be addressed to Tufan Turaci; tufanturaci@karabuk.edu.tr
}

Received 15 December 2015; Accepted 22 May 2016

Academic Editor: Manlio Gaudioso

Copyright ( 92016 T. Turaci and H. Aksan. This is an open access article distributed under the Creative Commons Attribution License, which permits unrestricted use, distribution, and reproduction in any medium, provided the original work is properly cited.

Let $G=(V(G), E(G))$ be an undirected simple connected graph. A network is usually represented by an undirected simple graph where vertices represent processors and edges represent links between processors. Finding the vulnerability values of communication networks modeled by graphs is important for network designers. The vulnerability value of a communication network shows the resistance of the network after the disruption of some centers or connection lines until a communication breakdown. The domination number and its variations are the most important vulnerability parameters for network vulnerability. Some variations of domination numbers are the 2-domination number, the bondage number, the reinforcement number, the average lower domination number, the average lower 2-domination number, and so forth. In this paper, we study the vulnerability of cycles and related graphs, namely, fans, $k$-pyramids, and $n$-gon books, via domination parameters. Then, exact solutions of the domination parameters are obtained for the above-mentioned graphs.

\section{Introduction}

Graph theory has become one of the most powerful mathematical tools in the analysis and study of the architecture of a network. Networks are important structures and appear in many different applications and settings. The study of networks has become an important area of multidisciplinary research involving computer science, mathematics, chemistry, social sciences, informatics, and other theoretical and applied sciences [1-3].

It is known that communication systems are often exposed to failures and attacks. So robustness of the network topology is a key aspect in the design of computer networks. The stability of a communication network, composed of processing nodes and communication links, is of prime importance to network designers. As the network begins losing links or nodes, eventually there is a loss in its effectiveness [4]. In the literature, various measures were defined to measure the robustness of network and a variety of graph theoretic parameters have been used to derive formulas to calculate network vulnerability. Graph vulnerability relates to the study of graph when some of its elements (vertices or edges) are removed. The measures of graph vulnerability are usually invariants that measure how the deletion of one or more network elements changes properties of the network $[5,6]$. The best known measure of reliability of a graph is its connectivity. The vertex (edge) connectivity is defined to be the minimum number of vertices (edges) whose deletion results in a disconnected or trivial graph [7]. Then the toughness [8], the integrity [9], the domination number $[10,11]$, the bondage number $[12,13]$, the 2-domination number [14], and the 2-bondage number [15] have been defined. Moreover, there are many graph theoretical parameters depending upon local damage for the graphs like the average lower independence number $[16,17]$, the average lower domination number $[17,18]$, the average connectivity [19], the average lower connectivity [20] and the average lower bondage number [6]. The average parameters have been found to be more useful in some circumstance than the corresponding measures based on worst-case situation [6].

A natural way to model the topology of a communications network is as a graph consisting of vertices and edges. In this paper, we consider simple finite undirected graphs by 
ignoring any variation in the type of edges. Let $G=$ $(V(G), E(G))$ be a simple undirected graph of order $n$. We begin by recalling some standard definitions that we need throughout this paper. For any vertex $v \in V(G)$, the open neighborhood of $v$ is $N_{G}(v)=\{u \in V \mid u v \in E(G)\}$ and closed neighborhood of $v$ is $N_{G}[v]=N_{G}(v) \cup\{v\}$. The degree of vertex $v$ in $G$ is denoted by $d_{G}(v)$, that is, the size of its open neighborhood. The maximum degree of $G$ is $\max \left\{d_{G}(v) \mid v \in\right.$ $V(G)\}$ and the minimum degree of $G$ is $\min \left\{d_{G}(v) \mid v \in\right.$ $V(G)$. The maximum and minimum degrees of a graph $G$ are denoted by $\Delta(G)$ and $\delta(G)$, respectively [11]. The graph $G$ is called $r$-regular graph if $d_{G}(v)=r$ for every vertex $v \in V(G)$. The vertex $v$ is called isolated vertex if $d_{G}(v)=0$. The null graph on $n$-vertices consists of $n$-isolated vertices with no edges. The join of graphs $G$ and $H$, denoted by $G \vee H$, is obtained from the disjoint union $G+H$ by adding the edges $\{v w \mid v \in V(G), w \in V(H)\}[21]$. We will use $\lfloor x\rfloor$ and $\lceil x\rceil$ for the largest integer not larger than $x$ and smallest integer not less than $x$, respectively.

Cycles and various related graphs have been studied for many reasons. Fans, wheels, pyramids, bipyramids, and $n$ cycle books are among such graphs. The definitions of these graphs will be given in Sections 3.2, 3.3, and 3.4.

Our aim in this paper is to consider the computing of the average lower domination number (ALDN) and the average lower 2-domination number (AL2DN) of some networks including cycles. In Section 2, definitions and well-known basic results have been given for ALDN and AL2DN, respectively. In Section 3, ALDN and AL2DN of some networks including cycles, namely, fans, $k$-pyramids, and $n$-gon books, have been determined.

\section{The Average Lower Domination Number Parameters and Basic Results}

A set $S \subseteq V(G)$ is a dominating set if every vertex in $V(G)-S$ is adjacent to at least one vertex in $S$. The minimum cardinality taken over all dominating sets of $G$ is called the domination number of $G$ and it is denoted by $\gamma(G)$ [10]. Moreover, a 2dominating set of a graph $G$ is a set $D \subseteq V(G)$ of vertices of graph $G$ such that every vertex of $V(G)-D$ has at least two neighbors in $D$. The 2-domination number of a graph $G$, denoted by $\gamma_{2}(G)$, is the minimum cardinality of a 2 dominating set of the graph $G[2,11,14]$.

In 2004, Henning introduced the concept of average domination and average independence [17]. The average lower domination number of a graph $G$, denoted by $\gamma_{\mathrm{av}}(G)$, is defined as $\gamma_{\mathrm{av}}(G)=(1 /|V(G)|) \sum_{v \in V(G)} \gamma_{v}(G)$, where the lower domination number, denoted by $\gamma_{v}(G)$, is the minimum cardinality of a dominating set of the graph $G$ that contains the vertex $v[17,18,22]$. In [23], the average lower 2domination number of a graph $G$ was defined. The AL2DN is defined by $\gamma_{2 \text { av }}(G)=(1 /|V(G)|) \sum_{v \in V(G)} \gamma_{2 v}(G)$, where the lower 2-domination number, denoted by $\gamma_{2 v}(G)$, of the graph $G$ relative to $v$ is the minimum cardinality of 2-dominating set in the graph $G$ that contains the vertex $v$. Moreover, Turaci showed that AL2DN is more sensitive than other vulnerability parameters, namely, connectivity, domination number, ALDN, and 2-domination number, in [23].
Theorem 1 (see [17]). For any graph $G$ of order $n$ with domination number $\gamma(G), \gamma_{a v}(G) \leq(\gamma(G)+1)-\gamma(G) / n$, with equality if and only if $G$ has a unique $\gamma(G)$-set.

Theorem 2 (see [17]). If $K_{1, n-1}$ is a star graph of order $n$, where $n \geq 3$, then $\gamma_{a v}\left(K_{1, n-1}\right)=(2 n-1) / n$.

Theorem 3 (see [17]). If $P_{n}$ is a path graph of order $n$, then

$$
\gamma_{a v}\left(P_{n}\right)= \begin{cases}\frac{n+2}{3}-\frac{2}{3 n}, & \text { if } n \equiv 2(\bmod 3) ; \\ \frac{n+2}{3}, & \text { otherwise. }\end{cases}
$$

Theorem 4 (see [17]). If $K_{n}$ is a complete graph of order $n$, then $\gamma_{a v}\left(K_{n}\right)=1$.

Observation 5. If $W_{n}$ is a wheel graph of order $n+1$, then $\gamma_{\mathrm{av}}\left(W_{n}\right)=(2 n+1) /(n+1)$.

Theorem 6 (see [15]). If $K_{n}$ is a complete graph of order $n$, where $n \geq 2$, then $\gamma_{2}\left(K_{n}\right)=2$.

Theorem 7 (see [15]). If $P_{n}$ is a path graph of order $n$, then $\gamma_{2}\left(P_{n}\right)=\lfloor n / 2\rfloor+1$.

Theorem 8 (see [15]). If $C_{n}$ is a cycle graph of order $n$, where $n \geq 3, \gamma_{2}\left(C_{n}\right)=\lfloor(n+1) / 2\rfloor$.

Theorem 9 (see [15]). If $W_{n}$ is a wheel graph of order $n+1$, where $n \geq 5$,

$$
\gamma_{2}\left(W_{n}\right)= \begin{cases}2, & \text { if } n=3,4 \\ 1+\left\lceil\frac{n}{3}\right\rceil, & \text { otherwise. }\end{cases}
$$

Theorem 10 (see [23]). Let $G$ be any connected graph of order $n$. If $\gamma_{2}(G)$-set is unique, then

$$
\gamma_{2 a v}(G)=\left(\gamma_{2}(G)+1\right)-\frac{\gamma_{2}(G)}{n} .
$$

Theorem 11 (see [23]). Let $G$ be any connected graph of order $n$. If $\delta(G) \geq 2$, then

$$
\gamma_{2}(G) \leq \gamma_{2 a v}(G) \leq\left(\gamma_{2}(G)+1\right)-\frac{\gamma_{2}(G)}{n}
$$

Theorem 12 (see [23]). Let $G$ be any connected graph of order $n$, where $n \geq 2$. Then,

$$
2 \leq \gamma_{2 a v}(G) \leq n-1+\frac{1}{n}
$$

Theorem 13 (see [23]). Let $G$ and $H$ be two connected graphs of order $n$ and $m$, respectively. If $n \geq 2$ and $m \geq 2$, then $\gamma_{2 a v}(G)+\gamma_{2 a v}(H) \geq \gamma_{2 a v}(G+H)$.

Theorem 14 (see [23]). Let $T$ be any connected tree of order $n$. IfT has s support vertices and $(n-s)$ leaf vertices, then $\gamma_{2 a v}(T) \geq$ $n-s+s / n$. 
Theorem 15 (see [23]). If $P_{n}$ is a path graph of order $n$, then

$$
\gamma_{2 a v}\left(P_{n}\right)= \begin{cases}\left\lfloor\frac{n}{2}\right\rfloor+2-\frac{\lfloor n / 2\rfloor+1}{n}, & \text { if } n \text { is odd } \\ \left\lfloor\frac{n}{2}\right\rfloor+1, & \text { if } n \text { is even. }\end{cases}
$$

Theorem 16 (see [23]). If $W_{n}$ is a wheel graph of order $n+1$, where $n \geq 5$, then

$$
\gamma_{2 a v}\left(W_{n}\right)=1+\left\lceil\frac{n}{3}\right\rceil
$$

Theorem 17 (see [23]). If $K_{n}$ is a complete graph of order $n$, where $n \geq 2$, then $\gamma_{2 a v}\left(K_{n}\right)=2$.

Theorem 18 (see [23]). If $K_{1, n-1}$ is a star graph of order $n$, where $n \geq 3$, then

$$
\gamma_{2 a v}\left(K_{1, n-1}\right)=n-1+\frac{1}{n}
$$

\section{Calculation of ALDN and AL2DN of Cycles and Related Networks}

\subsection{Cycles}

Theorem 19 (see [17]). If $C_{n}$ is a cycle graph of order $n$, then $\gamma_{a v}\left(C_{n}\right)=2$.

Theorem 20 (see [23]). If $C_{n}$ is a cycle graph of order $n$, then $\gamma_{2 a v}\left(C_{n}\right)=\lfloor(n+1) / 2\rfloor$.

\subsection{Fans}

Definition 21 (see [21]). If one joins a vertex of $C_{n}(n \geq 3)$ to all other vertices, the resulting graph is called a fan (also known as a shell), denoted by $F_{n}$. For $n=3$, we notice that $F_{3} \equiv C_{3}$. Fans can be described by the join operation $F_{n}=$ $P_{n-1}+v$, where $n \geq 3$. There is a vertex with $(n-1)$-degree, namely, $u$, in the graph $F_{n}$.

Theorem 22. Let $F_{n}$ be a fan of order $n$ and $n \geq 5$; then $\gamma_{2}\left(F_{n}\right)=\lceil(n+2) / 3\rceil$.

Proof. The 2-dominating set is formed by two ways in $F_{n}$.

Case 1. Let $D_{1}$ be a 2-dominating set and let $D_{1}$ include the vertex $u$. So, the vertex $u$ dominates vertices of $V\left(F_{n}\right)-\{u\}$ by once. Clearly, these $(n-1)$-vertices which are dominated once form the path $P_{n-1}$. Due to the fact that $\gamma\left(P_{n-1}\right)=\lceil(n-$ $1) / 3\rceil$, these $\lceil(n-1) / 3\rceil$ vertices must be taken to the set $D_{1}$. So, $\left|D_{1}\right|=1+\lceil(n-1) / 3\rceil=\lceil(n+2) / 3\rceil$ is obtained.

Case 2. Let $D_{2}$ be a 2-dominating set and let $D_{2}$ not include vertex $u$. So, the set $D_{2}$ must include vertices of subgraph path $P_{n-1}$. By Theorem 7, we have $\left|D_{2}\right|=1+\lceil(n-1) / 2\rceil=\lceil(n+$ $1) / 21$.
By Cases 1 and 2, $\left|D_{2}\right| \geq\left|D_{1}\right|$ is obtained for $n \geq 5$. As a result, we get $\gamma_{2}\left(F_{n}\right)=\lceil(n+2) / 3\rceil$.

Theorem 23. Let $F_{n}$ be a fan of order $n$ and $n \geq 3$; then $\gamma_{a v}\left(F_{n}\right)=(2 n-1) / n$.

Proof. By the definition of domination number and the structure of $F_{n}$, the dominating set of $F_{n}$ is unique and $\gamma\left(F_{n}\right)=$ 1 . By Theorem 1 , we get $\gamma_{a v}\left(F_{n}\right)=1+1-1 / n=(2 n-1) / n$.

Theorem 24. Let $F_{n}$ be a fan of order $n$ and $n \geq 5$; then

$$
\gamma_{2 a v}\left(F_{n}\right)=\frac{1}{n}\left[\left\lceil\frac{(n+2)}{3}\right]+(n-1)\left(\gamma_{a v}\left(P_{n-1}\right)+1\right)\right] .
$$

Proof. When $\gamma_{2 v}\left(F_{n}\right)$ is calculated for all vertices in $F_{n}$, the vertices in three cases should be examined.

Case 1. For the vertex $u$, the 2 -dominating set must include the vertex $u$ by Theorem 22. The rest of the proof of this case is similar to Case 1 of Theorem 22. So, we get $\gamma_{2 u}\left(F_{n}\right)=1+$ $\lceil(n-1) / 3\rceil=\lceil(n+2) / 3\rceil$.

Case 2. For all vertices $v_{i} \in V\left(F_{n}\right)-\{u\}$ which forms the graph $P_{n-1}$. We know that $\sum_{i=1}^{n-1} \gamma_{v_{i}}\left(F_{n}\right)=(n-1) \gamma_{\mathrm{av}}\left(P_{n-1}\right)$ by the definition of ALDN. Furthermore, we know that the vertex $u$ must be in the 2-dominating set. So, we have $\sum_{i=1}^{n-1} \gamma_{2 v_{i}}=$ $(n-1) \gamma_{\mathrm{av}}\left(P_{n-1}\right)+(n-1)$ for the sum of the lower 2domination number of all vertices $v_{i} \in V\left(F_{n}\right)-\{u\}$. As a result, $\sum_{i=1}^{n-1} \gamma_{2 v_{i}}\left(F_{n}\right)=(n-1)\left(\gamma_{\mathrm{av}}\left(P_{n-1}\right)+1\right)$ is obtained.

By Cases 1 and 2, we get

$$
\begin{aligned}
\gamma_{2 \mathrm{av}}\left(F_{n}\right) & =\frac{1}{\left|V\left(F_{n}\right)\right|}\left(\gamma_{2 u}\left(F_{n}\right)+\sum_{i=1}^{n-1} \gamma_{2 v_{i}}\left(F_{n}\right)\right) \\
& =\frac{1}{n}\left[\left\lceil\frac{(n+2)}{3}\right\rceil+(n-1)\left(\gamma_{\mathrm{av}}\left(P_{n-1}\right)+1\right)\right] .
\end{aligned}
$$

Remark 25. Let $F_{n}$ be a fan of order $n$ and $n \geq 5$; then

$$
\begin{aligned}
& \gamma_{2 \mathrm{av}}\left(F_{n}\right) \\
& = \begin{cases}\frac{\left(n^{2}+3 n-3\right) / 3+\lceil(n-1) / 3\rceil}{n}, & \text { if } n \equiv 0(\bmod 3) ; \\
\frac{\left(n^{2}+3 n-1\right) / 3+\lceil(n-1) / 3\rceil}{n}, & \text { otherwise. }\end{cases}
\end{aligned}
$$

Proof. This is clear from Theorems 3 and 24.

\section{3. k-Pyramids}

Definition 26 (see [21]). The join graph $C_{n} \vee N_{k}(n \geq 3, k \geq$ 1 ), where $N_{k}$ is the null graph of order $k$, is called a $k$-pyramid and is denoted by $k P(n)$. The 2-pyramid $C_{n} \vee N_{2}$ is called bipyramid and is denoted by $\operatorname{BP}(n)$. The 1-pyramid $C_{n} \vee N_{1}$ is the wheel graph $W_{n}$. 
Theorem 27. Let $B P(n)$ be a bipyramid of order $n+2$ and $n \geq$ 7 ; then $\gamma(B P(n))=2$.

Proof. Because $\Delta(\mathrm{BP}(n))<n+1$, the domination number $\gamma(\mathrm{BP}(n))$ is greater than 1 . Let $v_{1}$ and $v_{2}$ be vertices whose degrees are $n$, and let $D$ be a dominating set. The dominating set $D$ is formed by 3 cases.

Case 1. Let $v_{1}, v_{2} \in D$. Due to $N_{\mathrm{BP}(n)}\left[v_{1}, v_{2}\right]=V(\mathrm{BP}(n))$, the set $D$ is a dominating set.

Case 2. Let $v_{1} \in D$ and let $u_{i}$ be any vertex of $C_{n}$. Due to the fact that $N_{\mathrm{BP}(n)}\left[v_{1}, u_{i}\right]=V(\mathrm{BP}(n))$, the set $D$ is a dominating set.

Case 3. Let $v_{1}, v_{2} \notin D$. Then, the set $D$ includes only vertices of $C_{n}$. So, we have $|D| \geq\lceil n / 3\rceil$.

By Cases 1, 2, and 3, $\gamma(\mathrm{BP}(n))=2$ is obtained.

Theorem 28. Let $B P(n)$ be a bipyramid of order $n+2$ and $n \geq 7$; then

$$
\gamma_{a v}(B P(n))=2
$$

Proof. When $\gamma_{v}(\mathrm{BP}(n))$ is calculated for all vertices in the graph $\mathrm{BP}(n)$, the vertices in two cases should be examined. Let $v_{1}$ and $v_{2}$ be vertices whose degrees are $n$.

Case 1. For the vertices $v_{1}$ and $v_{2}$, we know that the set $\left\{v_{1}, v_{2}\right\}$ dominates all vertices of $V(\operatorname{BP}(n))-\left\{v_{1}, v_{2}\right\}$. So, we get $\gamma_{v_{1}}(\mathrm{BP}(n))=\gamma_{v_{2}}(\mathrm{BP}(n))=2$.

Case 2. For all vertices $v_{i} \in V(\mathrm{BP}(n))-\left\{v_{1}, v_{2}\right\}$, by the definition of lower domination number and Case 2 of Theorem 27, we have $\gamma_{v_{i}}(\mathrm{BP}(n))=2$ for all vertices $v_{i} \in V(\mathrm{BP}(n))-\left\{v_{1}, v_{2}\right\}$.

By Cases 1 and 2, we get $\gamma_{\mathrm{av}}(\mathrm{BP}(n))=2$.

Theorem 29. Let $B P(n)$ be a bipyramid of order $n+2$ and $n \geq 3$; then $\gamma_{2}(B P(n))=2$. Furthermore, the 2-dominating set of $B P(n)$ is unique.

Proof. Let $u_{i}$ be the vertices of $C_{n}$, where $1 \leq i \leq n$, and let $v_{j}$ be vertices of the graph $\overline{K_{2}}$, where $1 \leq j \leq 2$. There are 3 cases while forming 2-dominating set.

Case 1. Let $D_{1}$ be a 2-dominating set of $\mathrm{BP}(n)$. Furthermore, the set $D_{1}$ contains vertices $v_{1}$ and $v_{2}$. Clearly, $N_{\mathrm{BP}(n)}\left[v_{1}, v_{2}\right]=$ $V(\mathrm{BP}(n))$. So, $\left|D_{1}\right|=2$ is obtained.

Case 2. Let $D_{2}$ be any 2-dominating set, and the set $D_{2}$ contains $v_{1}$ or $v_{2}$. So, the set $D_{2} 1$-dominates all vertices of $C_{n}$. By the definition of $\gamma\left(C_{n}\right)$, if we add $\lceil n / 3\rceil$ vertices to the set $D_{2}$, then all of $C_{n}$ graph's vertices are 2-dominated. So, $\lceil n / 3\rceil \geq 2$ for $n \geq 5$. Hence, $\left|D_{2}\right|=1+\lceil n / 3\rceil$ is obtained.

Case 3. Let $D_{3}$ be any 2-dominating set. Moreover, the set $D_{3}$ contains only vertices $u_{i}$ of $C_{n}$. It is clear that the set $D_{3}$ is the 2-dominating set of $C_{n}$. By Theorem 8 , we have $\left|D_{3}\right|=$ $\lfloor(n+1) / 2\rfloor$.

By Cases 1, 2, and 3 we get $\left|D_{1}\right|=2,\left|D_{2}\right| \geq 3$, and $\left|D_{3}\right| \geq$ 3 for $n \geq 5$. As a result, by the definition of 2-domination number, we have $\gamma_{2}(\mathrm{BP}(n))=2$ and this 2 -dominating set is unique.

Theorem 30. Let $B P(n)$ be a bipyramid of order $n+2$ and $n \geq 3$; then

$$
\gamma_{2 a v}(B P(n))=\frac{3 n+4}{n+2} .
$$

Proof. Since the 2-dominating set is unique, we have $\gamma_{2 \mathrm{av}}(\mathrm{BP}(n))=2+1-2 /(n+2)=(3 n+4) /(n+2)$ by Theorem 10.

Theorem 31. For the 3-pyramid $3 P(n)$ with $n \geq 7, \gamma_{2}(3 P(n))=$ 3.

Proof. There are 3 vertices whose degrees are $n$ in the graph $3 P(n)$ and they are shown by $u_{1}, u_{2}$, and $u_{3}$. We have four cases while 2-dominating set is forming.

Case 1. Let $D_{1}$ be any 2-dominating set of $3 P(n)$ and let $D_{1}$ contain vertices $u_{1}, u_{2}$, and $u_{3}$. It is clear that $N_{3 P(n)}\left[u_{1}, u_{2}, u_{3}\right]=V(3 P(n))$ is obtained. So, the set $D_{1}$ is 2-dominating set of $3 P(n)$. Thus, $\left|D_{1}\right|=3$ is obtained.

Case 2. Let $D_{2}$ be any 2-dominating set of $3 P(n)$ and let set $D_{2}$ include any two vertices of the vertices $u_{1}, u_{2}$, and $u_{3}$. These two vertices are 2-dominated by the vertices of $C_{n}$. If the remaining vertex is added to $D_{2}$ set, it is similar to set $D_{1}$ and all vertices of $3 P(n)$ are 2 -dominated. If the remaining vertex is not added to $D_{2}$ set, two vertices of $V(3 P(n))$ $\left\{u_{1}, u_{2}, u_{3}\right\}$ must be added to the set $D_{2}$. Thus, $\left|D_{2}\right| \geq 3$ is obtained.

Case 3. Let $D_{3}$ be any 2-dominating set of $3 P(n)$ and let the set $D_{3}$ include any one vertex of $u_{1}, u_{2}$, and $u_{3}$. So, the vertices of $C_{n}$ are dominated once by the set $D_{3}$. Since the set $D_{3}$ does not include the remaining two vertices of the vertices $u_{1}, u_{2}$, and $u_{3},\lceil n / 3\rceil$ vertices of $C_{n}$ must be taken to set $D_{3}$ to 2-dominate all vertices of $C_{n}$ and the remaining two vertices of the vertices $u_{1}, u_{2}$, and $u_{3}$. Because $n \geq 7$, we have $\left|D_{3}\right|=\lceil n / 3\rceil+1$.

Case 4. Let $D_{4}$ be any 2-dominating set of $3 P(n)$ and let the set $D_{4}$ not include any vertices of $u_{1}, u_{2}$, and $u_{3}$. So, we must add vertices of the graph $C_{n}$ to set $D_{4}$. We know that $\gamma_{2}\left(C_{n}\right)=$ $\lfloor(n+1) / 2\rfloor$ by Theorem 8. Thus, $\left|D_{4}\right| \geq\lfloor(n+1) / 2\rfloor$ is obtained.

By Cases $1,2,3$, and 4 we get $\left|D_{1}\right|=3,\left|D_{2}\right| \geq 3,\left|D_{3}\right| \geq$ $\lceil n / 3\rceil+1$, and $\left|D_{4}\right| \geq\lfloor(n+1) / 2\rfloor$ for $n \geq 7$. As a result we have $\gamma_{2}(3 P(n))=3$ by the definition of 2 -domination number and this 2-dominating set is unique.

Theorem 32. For the 3-pyramid $3 P(n)$ with $n \geq 7$, $\gamma_{2 a v}(3 P(n))=(4 n+9) /(n+3)$. 
Proof. Since the 2-dominating set is unique, we have $\gamma_{2 \mathrm{av}}(3 P(n))=3+1-3 /(n+3)=(4 n+9) /(n+3)$ by Theorem 10.

Theorem 33. Let $k P(n)$ be a $k$-pyramid with $k \geq 4$ and $n \geq 7$; then $\gamma_{2}(k P(n))=4$.

Proof. The vertices of $C_{n}$ are denoted by $v_{i}$, where $i \in$ $\{1, \ldots, n\}$, and the remaining $k$-vertices are denoted by $u_{j}$, where $j \in\{1, \ldots, k\}$. We have three cases while 2-dominating set is forming.

Case 1. Let $D_{1}$ be any 2-dominating set of the graph $k P(n)$ and let $D_{1}$ contain only vertices $u_{j}$, where $j \in\{1, \ldots, k\}$. It is clear that set $\left\{u_{1}, u_{2} \ldots, u_{j}\right\} 2$-dominated all vertices of $k P(n)$. So, $\left|D_{1}\right|=k$ and $\left|D_{1}\right| \geq 4$ are obtained.

Case 2. Let $D_{2}$ be any 2-dominating set of $k P(n)$ and let $D_{2}$ contain only vertices of $v_{i}$, where $i \in\{1, \ldots, n\}$. By Theorem 8 , we have $\left|D_{2}\right|=\lfloor(n+1) / 2\rfloor$. As a result, $\left|D_{2}\right| \geq 4$ is obtained.

Case 3. Let $D_{3}$ be any 2-dominating set of $k P(n)$ and let $D_{3}$ contain any vertex of $u_{j}$ and $v_{i}$, where $j \in\{1, \ldots, k\}$ and $i \in$ $\{1, \ldots, n\}$. Let $D_{3}=\left\{u_{1}, v_{1}\right\}$. Let $=\left\{u_{j} \mid 1 \leq j \leq k\right\}$. The vertex $u_{1}$ dominates vertices of $V\left(C_{n}\right)-\left\{u_{1}\right\}$ by once and the vertex $v_{1}$ dominates vertices of $U-\left\{v_{1}\right\}$ by once. If any vertex of $v_{i}$ and any vertex of $u_{j}$ are added to set $D_{3}$, then $D_{3}$ will be 2 -dominating set of the graph $k P(n)$. Hence, $\left|D_{3}\right|=4$ is obtained.

By Cases 1,2 , and 3 we get $\left|D_{1}\right| \geq 4,\left|D_{2}\right| \geq 4$, and $\left|D_{3}\right|=$ 4 for $n \geq 7$. As a result, by the definition of 2-domination number, we have $\gamma_{2}(k P(n))=4$.

Theorem 34. Let $k P(n)$ be a $k$-pyramid with $k \geq 4$ and $n \geq 7$; then $\gamma_{2 a v}(k P(n))=4$.

Proof. By Theorem 33, we have the lower 2-domination number as 4 for all vertices of $k P(n)$. Thus, $\gamma_{2 \text { av }}(k P(n))=4$ is obtained.

\section{4. n-Gon Books}

Definition 35 (see [21]). When $k$ copies of $C_{n}(n \geq 3)$ share a common edge, they will form an $n$-gon book of $k$ pages and are denoted by $B(n, k)$. The degree set of $B(n, k)$ is $\{2, k+1\}$. Therefore, the vertices of $B(n, k)$ are of two kinds: vertices of degree 2 , which will be referred to as minor vertices, and vertices of degree $k+1$, which will be referred to as major vertices. The minor vertices of $B(n, k)$ are labeled $v_{11}, v_{12} \ldots, v_{1 j}, v_{21}, v_{22}, \ldots, v_{2 j}, \ldots, v_{k 1}, v_{k 2}, \ldots, v_{k j}$, that is $v_{i j}$, where $i \in\{1, \ldots, k\}$ and $j \in\{1, \ldots, n-2\}$.

Theorem 36. Let $B(n, k)$ be an $n$-gon book and $n \geq 5$; then

$$
\gamma(B(n, k))= \begin{cases}k\left\lceil\frac{n-4}{3}\right\rceil+2, & n \equiv 1(\bmod 3) \\ k\left\lceil\frac{n-3}{3}\right\rceil+1, & \text { otherwise. }\end{cases}
$$

Proof. Let $u_{1}$ and $u_{2}$ be two major vertices. We have three cases depending on the dominating set of $B(n, k)$ that are including major vertices or not.

Case 1. Let $D_{1}$ be a dominating set, and let $D_{1}$ include two major vertices. The set $\left\{u_{1}, u_{2}\right\}$ dominates $2 k$-vertices from all $C_{n}$. So, there are $(n-4)$-vertices which are not dominated from each $C_{n}$. Then the $k$-distinct path $P_{n-4}$ is obtained by these minor vertices. Thus, $\left|D_{1}\right|=k\lceil(n-4) / 3\rceil+2$ is obtained.

Case 2. Let $D_{2}$ be a dominating set, and let $u_{1} \in D_{2}$ (or $\left.u_{2} \in D_{2}\right)$. The vertex $u_{1}$ dominates the vertex $u_{2}$ and $k$ vertices which are adjacent to the vertex $u_{1}$ from all $C_{n}$. Then the remaining $(n-3)$-vertices are not dominated from each $C_{n}$. So, the $k$-distinct path $P_{n-3}$ is obtained by these minor vertices. As a result, $\left|D_{2}\right|=k\lceil(n-4) / 3\rceil+1$ is obtained.

Case 3. Let $D_{3}$ be a dominating set, and let $\left\{u_{1}, u_{2}\right\} \notin D_{3}$. Since the set $D_{3}$ includes only minor vertices, we have two subcases depending on $n$.

Case 3.1. Let $n \equiv 0,1(\bmod 3)$. Due to the structure of $B(n, k)$, the set $D_{3}$ must include $\lceil(n-2) / 3\rceil$-vertices from each $C_{n}$. So, the whole vertices of $B(n, k)$ are dominated. As a result we have $\left|D_{3}\right|=k\lceil(n-2) / 3\rceil$.

Case 3.2. Let $n \equiv 2(\bmod 3)$. Due to the structure of $B(n, k)$, two minor vertices which are neighbors to the major vertices must be taken to the set $D_{3}$ from any graph $C_{n}$. Furthermore, the remaining $(k-1)(n-2)$-vertices are not dominated in the graph $B(n, k)$. Since $(k-1)\lceil(n-2) / 3\rceil$-vertices must be taken to the set $D_{3},\left|D_{3}\right|=2+\lceil(n-6) / 3\rceil+(k-1)\lceil(n-2) / 3\rceil$ is obtained.

By Cases 3.1 and 3.2, we get

$$
\begin{aligned}
& \left|D_{3}\right| \\
& = \begin{cases}2+\left\lceil\frac{n-6}{3}\right\rceil+(k-1) \cdot\left\lceil\frac{n-2}{3}\right\rceil, & n \equiv 2(\bmod 3) ; \\
k \cdot\left\lceil\frac{n-2}{3}\right\rceil, & \text { otherwise. }\end{cases}
\end{aligned}
$$

Clearly, if $n \equiv 0(\bmod 3)$, then we have $\left|D_{3}\right|>\left|D_{1}\right|>\left|D_{2}\right|$, if $n \equiv 1(\bmod 3)$, then we have $\left|D_{2}\right|>\left|D_{3}\right|>\left|D_{1}\right|$, and if $n \equiv 2(\bmod 3)$, then we have $\left|D_{1}\right|>\left|D_{3}\right|=\left|D_{2}\right|$. As a result, we get

$$
\gamma B(n, k)= \begin{cases}k\left\lceil\frac{n-4}{3}\right\rceil+2, & n \equiv 1(\bmod 3) \\ k\left\lceil\frac{n-3}{3}\right\rceil+1, & \text { otherwise. }\end{cases}
$$

Thus the proof is completed.

Theorem 37. Let $B(n, k)$ be an $n$-gon book and $n \geq 5$; then 


$$
\begin{aligned}
\gamma_{a v}(B(n, k)) & \left\{\begin{array}{lr}
k\left\lceil\frac{n-3}{3}\right\rceil+1, & \text { if } n \equiv 2(\bmod 3) ; \\
3+k\left\lceil\frac{n-4}{3}\right\rceil-\frac{2+k\lceil(n-4) / 3\rceil}{k(n-2)+2}, & \text { if } n \equiv 1(\bmod 3) ; \\
\frac{1}{k(n-2)+2}\left(\left[(n-2)(k+2)-k\left\lceil\frac{n}{3}\right\rceil\right]\left(1+k\left\lceil\frac{n-3}{3}\right\rceil\right)+k\left\lceil\frac{n}{3}\right\rceil\left(2+k\left\lceil\frac{n-3}{3}\right\rceil\right)\right), & \text { if } n \equiv 0(\bmod 3) .
\end{array}\right.
\end{aligned}
$$

Proof. While finding lower domination number of all vertices of $B(n, k)$, we have three cases depending on $n$.

Case 1 . Let $n \equiv 1(\bmod 3)$, and let $D$ be a dominating set. Clearly, the set $D$ is a unique dominating set. By Theorem 10, we have

$$
\gamma_{\mathrm{av}}(B(n, k))=3+k\left\lceil\frac{n-4}{3}\right\rceil-\frac{2+k\lceil(n-4) / 3\rceil}{k(n-2)+2} .
$$

Case 2. Let $n \equiv 2(\bmod 3)$, and let $D$ be a dominating set. By Theorem 36, the set $D$ is formed by two ways. Clearly, $\gamma_{v_{i}}(B(n, k))=\lceil(n-3) / 3\rceil+1$ is obtained for every $v_{i} \epsilon$ $V(B(n, k))$. As a result, we have $\gamma_{\mathrm{av}}(B(n, k))=1+k\lceil(n-3) / 3\rceil$.

Case 3 . Let $n \equiv 0(\bmod 3)$, and let $D$ be a dominating set. By Case 2 of Theorem 36, we know that the set $D$ includes either the vertex $u_{1}$ or the vertex $u_{2}$. Let $S=\left\{v_{i j} \mid i \in\{1, \ldots, k\} \wedge\right.$ $j \in\{1,4, \ldots, n-2\}\}$. Clearly, the set $D$ contains vertices of $V(B(n, k))-S$ by Theorem 36. Thus, we have $\gamma_{v_{i}}(B(n, k))=$ $1+k\lceil(n-3) / 3\rceil$ for every $v_{i} \in V(B(n, k))-S$. Furthermore, we have $\gamma_{v_{i}^{*}}(B(n, k))=2+k\lceil(n-3) / 3\rceil$ for every $v_{i}^{*} \in S$. Thus,

$$
\begin{aligned}
\gamma_{\mathrm{av}} & (B(n, k)) \\
= & \frac{1}{|V(B(n, k))|}\left(\sum_{v_{i} \in V(B(n, k))-S} \gamma_{v_{i}}(B(n, k))\right. \\
& \left.+\sum_{v_{i}^{*} \in S} \gamma_{v_{i}^{*}}(B(n, k))\right) \\
& =\frac{1}{k(n-2)+2}\left(\left[(n-2)(k+2)-k\left\lceil\frac{n}{3}\right\rceil\right]\right. \\
& \left.\cdot\left(1+k\left\lceil\frac{n-3}{3}\right\rceil\right)+k\left\lceil\frac{n}{3}\right\rceil\left(2+k\left\lceil\frac{n-3}{3}\right\rceil\right)\right) .
\end{aligned}
$$

By Cases 1, 2, and 3 the proof is completed.
Theorem 38. Let $B(n, k)$ be an $n$-gon book and $n \geq 5$; then

$$
\gamma_{2}(B(n, k))= \begin{cases}k\left(\left\lfloor\frac{n+1}{2}\right\rfloor-1\right)+1, & \text { if } n \text { is even } \\ k\left(\left\lfloor\frac{n-4}{2}\right\rfloor+1\right)+2, & \text { if } n \text { is odd } .\end{cases}
$$

Proof. Let $u_{1}$ and $u_{2}$ be two major vertices. We have two cases depending on $n$.

Case 1. Let $n$ be even, and let $D$ be 2 -dominating set. We know that the set $D$ contains either the vertex $u_{1}$ or the vertex $u_{2}$ by the definition of $B(n, k)$ and Theorem 8 . Thus, $(\lfloor(n+1) / 2\rfloor-1)$ vertices of each $C_{n}$ must be taken to the set $D$. Since there is $k$ graph $C_{n}$, we have $|D|=k(\lfloor(n+1) / 2\rfloor-1)+1$. So, $\gamma_{2}(B(n, k))=$ $k(\lfloor(n+1) / 2\rfloor-1)+1$ is obtained.

Case 2. Let $n$ be odd. We have two subcases depending on the 2-dominating set of $B(n, k)$ that are including major vertices or not.

Case 2.1. Let $D_{1}$ be 2-dominating set, and let $D_{1}$ include any major vertex. Clearly, the set $D$ must include two major vertices in this subcase by Theorem 8 . So, the set $\left\{u_{1}, u_{2}\right\}$ dominates $2 k$-vertices from all $C_{n}$. Then, $(n-4)$-vertices are not dominated from each $C_{n}$. Thus, the $k$-distinct path $P_{n-4}$ is obtained by these minor vertices. As a result, $\left|D_{1}\right|=k(\lfloor(n-$ $4) / 2\rfloor+1)+2$ is obtained.

Case 2.2. Let $D_{2}$ be 2-dominating set, and let $\left\{u_{1}, u_{2}\right\} \notin D_{2}$. Clearly, the set $D_{2}$ includes $(\lfloor(n-2) / 2\rfloor+1)$-vertices of $V\left(C_{n}\right)-$ $\left\{u_{1}, u_{2}\right\}$ of each $C_{n}$ by Theorem 7 . Thus, $\left|D_{2}\right|=k(\lfloor(n-2) / 2\rfloor+$ 1) is obtained.

By Cases 2.1 and $2.2\left|D_{1}\right|=\left|D_{2}\right|$ for $k=2$ and $n \geq 5$. Then we have $\left|D_{1}\right|<\left|D_{2}\right|$ for $k \geq 3$ and $n \geq 5$. Furthermore, 2-dominating set is unique for $k \geq 3$ and $n \geq 5$. As a result, $\gamma_{2}(B(n, k))=k(\lfloor(n-4) / 2\rfloor+1)+2$ is obtained.

By Cases 1 and 2, the proof is completed.

Theorem 39. Let $B(n, k)$ be an $n$-gon book, where $n \geq 3$ and $k \geq 3$; then

$$
\gamma_{2 a v}(B(n, k))= \begin{cases}k\left(\left\lfloor\frac{n+1}{2}\right\rfloor-1\right)+1, & \text { if } n \text { is even } \\ k\left(\left\lfloor\frac{n-4}{2}\right\rfloor+1\right)+3-\frac{k(\lfloor(n-4) / 2\rfloor+1)+2}{k(n-2)+2}, & \text { if } n \text { is odd } .\end{cases}
$$


Proof. Let $u_{1}$ and $u_{2}$ be two major vertices. We have two cases depending on $n$.

Case 1. Let $n$ be even. We have that the 2-dominating set including the vertex $u_{1}$ is 2 -dominating set of any $C_{n}$. Since the vertex $u_{1}$ is common vertex of each $C_{n}$ and by Theorem 8 , we get $\gamma_{2 u_{1}}(B(n, k))=k(\lfloor(n+1) / 2\rfloor-1)+1$. Similarly, we get $\gamma_{2 u_{2}}(B(n, k))=k(\lfloor(n+1) / 2\rfloor-1)+1$. Let $v_{i 1}$ be minor vertex which is neighbor to the vertex $u_{1}$ of any $C_{n}$, and let $v_{i(n-2)}$ be minor vertex which is neighbor to the vertex $u_{2}$ in the same graph $C_{n}$. It is easy to see that the 2-dominating set including the vertex $v_{i 1}$ is the 2-dominating set including the vertex $u_{2}$ by Theorem 8 . Similarly, the 2 -dominating set including the vertex $v_{i(n-2)}$ is the 2-dominating set including the vertex $u_{1}$. Thus, $\gamma_{2 v_{i 1}}(B(n, k))=\gamma_{2 v_{i(n-2)}}(B(n, k))=k(\lfloor(n+$ $1) / 2\rfloor-1)+1$ is obtained. Furthermore, we get $\gamma_{2 v_{i j}}(B(n, k))=$ $k(\lfloor(n+1) / 2\rfloor-1)+1$ for every $v_{i j} \in V(B(n, k))-\left\{u_{1}, u_{2}\right\}$, where $i \in\{1, \ldots, k\}$ and $j \in\{1, \ldots, n-2\}$. As a result, $\gamma_{2 \mathrm{av}}(B(n, k))=k(\lfloor(n+1) / 2\rfloor-1)+1$ is obtained.

Case 2. Let $n$ be odd. We have two subcases depending on $k$.

Case 2.1. Let $k=2$. We know that 2-dominating set is formed by two ways in Case 2 of Theorem 38. Clearly, $\gamma_{2 v}(B(n, k))=$ $k(\lfloor(n-4) / 2\rfloor+1)+2$ is obtained for every vertex $v \in V(B(n, k))$. As a result, $\gamma_{2 \text { av }}(B(n, k))=k(\lfloor(n-4) / 2\rfloor+1)+2$ is obtained.

Case 2.2. Let $k \geq 3$. We know that 2 -dominating set is unique by Case 2 of Theorem 38. So, $\gamma_{2 \mathrm{av}}(B(n, k))=k(\lfloor(n-4) / 2\rfloor+$ $1)+3-((k(\lfloor(n-4) / 2\rfloor+1)+2) / k(n-2)+2)$ is obtained by Theorem 10 .

By Cases 1 and 2 the proof is completed.

Theorem 40. Let $B(n, k)$ be an $n$-gon book, where $n \geq 3$ and $k=2$; then

$$
\begin{aligned}
\gamma_{2 a v}(B(n, k)) \\
=\left\{\begin{array}{l}
k\left(\left\lfloor\frac{n+1}{2}\right\rfloor-1\right)+1, \quad \text { if } n \text { is even; } \\
k\left(\left\lfloor\frac{n-4}{2}\right\rfloor+1\right)+2, \quad \text { if } n \text { is odd. }
\end{array}\right.
\end{aligned}
$$

Proof. The proof directly comes from Theorem 39.

\section{Conclusion}

In this study, a new defined graph theoretical parameter, namely, the average lower 2-domination number and the average lower domination number, has been studied for the network vulnerability. Additionally, the stability of popular interconnection networks including cycles has been studied and their domination numbers, 2-domination numbers, average lower domination numbers, and average lower domination 2-numbers, have been computed. These networks have been modeled with the fans, the $k$-pyramids, and the $n$-gon books.

\section{Competing Interests}

The authors declare that they have no competing interests.

\section{Acknowledgments}

The authors would like to thank Professor Dr. Manlio Gaudioso for the constructive suggestions and comments that improve the quality of this paper.

\section{References}

[1] I. Mishkovski, M. Biey, and L. Kocarev, "Vulnerability of complex networks," Communications in Nonlinear Science and Numerical Simulation, vol. 16, no. 1, pp. 341-349, 2011.

[2] K. T. Newport and P. K. Varshney, "Design of survivable communications networks under performance constraints," IEEE Transactions on Reliability, vol. 40, no. 4, pp. 433-440, 1991.

[3] T. Turaci and M. Ökten, "The edge eccentric connectivity index of hexagonal cactus chains," Journal of Computational and Theoretical Nanoscience, vol. 12, no. 10, pp. 3977-3980, 2015.

[4] T. Turaci and M. Okten, "Vulnerability of Mycielski graphs via residual closeness," Ars Combinatoria, vol. 118, pp. 419-427, 2015.

[5] E. Aslan, "Neighbour isolated scattering number of graphs," ScienceAsia, vol. 41, no. 6, pp. 423-431, 2015.

[6] T. Turaci, "On the average lower bondage number a graph," RAIRO-Operations Research, 2015.

[7] H. Frank and I. T. Frisch, "Analysis and design of survivable networks," IEEE Transactions on Communications, vol. 18, pp. 501-519, 1970.

[8] V. Chvátal, “Tough graphs and Hamiltonian circuits," Discrete Mathematics, vol. 5, pp. 215-228, 1973.

[9] C. A. Barefoot, R. Entringer, and H. Swart, "Vulnerability in graphs-a comparative survey," Journal of Combinatorial Mathematics and Combinatorial Computing, vol. 1, pp. 13-22, 1987.

[10] D. Bauer, F. Harary, J. Nieminen, and C. L. Suffel, "Domination alteration sets in graphs," Discrete Mathematics, vol. 47, pp. 153$161,1983$.

[11] T. W. Haynes, S. T. Hedeniemi, and P. J. Slater, Fundamentals of Domination in Graphs, Advanced Topic, Marcel Dekker, New York, NY, USA, 1998.

[12] A. Aytaç, T. Turaci, and Z. N. Odabaş, "On the bondage number of middle graphs," Mathematical Notes, vol. 93, no. 5-6, pp. 795801, 2013.

[13] A. Aytaç, Z. N. Odabas, and T. Turaci, “The bondage number of some graphs," Comptes Rendus de Lacademie Bulgare des Sciences, vol. 64, no. 7, pp. 925-930, 2011.

[14] M. Chellali, "Bounds on the 2-domination number in cactus graphs," Opuscula Mathematica, vol. 26, no. 1, pp. 5-12, 2006.

[15] M. Krzywkowski, "2-bondage in graphs," International Journal of Computer Mathematics, vol. 90, no. 7, pp. 1358-1365, 2013.

[16] A. Aytaç and T. Turaci, "Vertex vulnerability parameter of gear graphs," International Journal of Foundations of Computer Science, vol. 22, no. 5, pp. 1187-1195, 2011.

[17] M. A. Henning, "Trees with equal average domination and independent domination numbers," Ars Combinatoria, vol. 71, pp. 305-318, 2004.

[18] V. Aytaç, "Average lower domination number in graphs," Comptes Rendus de Lacademie Bulgare des Sciences, vol. 65, no. 12, pp. 1665-1674, 2012. 
[19] L. W. Beineke, O. R. Oellermann, and R. E. Pippert, "The average connectivity of a graph," Discrete Mathematics, vol. 252, no. 1-3, pp. 31-45, 2002.

[20] E. Aslan, "The average lower connectivity of graphs," Journal of Applied Mathematics, vol. 2014, Article ID 807834, 4 pages, 2014.

[21] Z. N. Odabaş and A. Aytaç, "Residual closeness in cycles and related networks," Fundamenta Informaticae, vol. 124, no. 3, pp. 297-307, 2013.

[22] M. Blidia, M. Chellali, and F. Maffray, "On average lower independence and domination numbers in graphs," Discrete Mathematics, vol. 295, no. 1-3, pp. 1-11, 2005.

[23] T. Turaci, "The concept of vulnerability in graphs and average lower 2-domination number," in Proceedings of the 28th National Mathematic Conference, Antalya, Turkey, September 2015. 


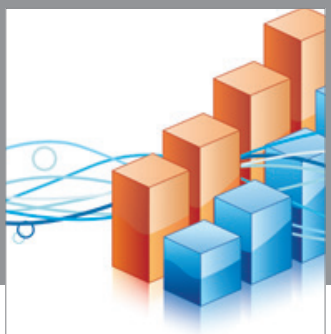

Advances in

Operations Research

vatem alat4

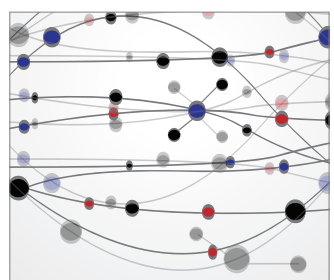

\section{The Scientific} World Journal
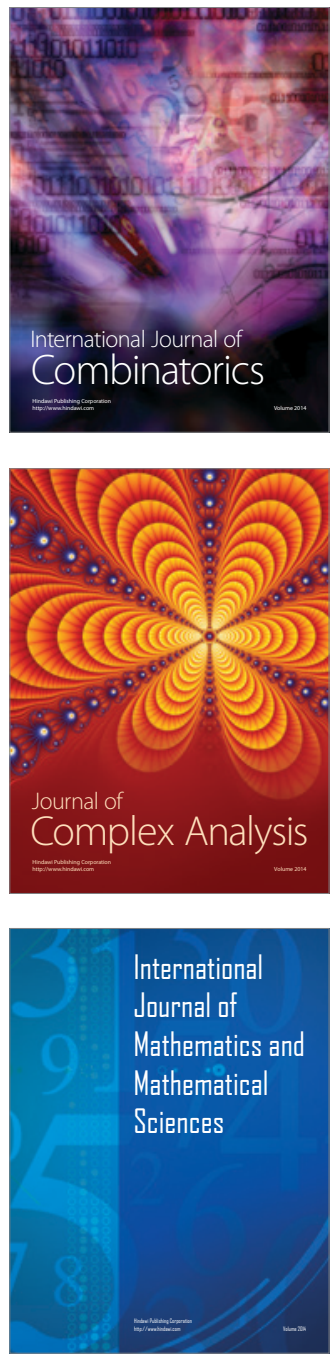
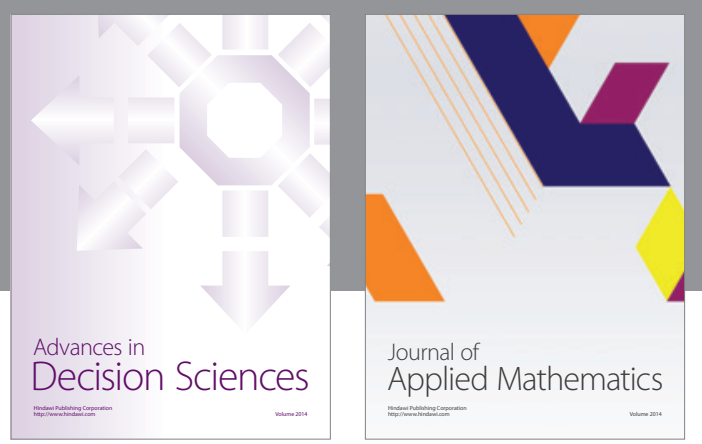

Algebra

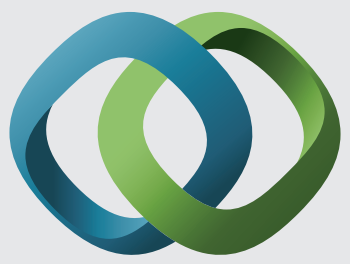

\section{Hindawi}

Submit your manuscripts at

http://www.hindawi.com
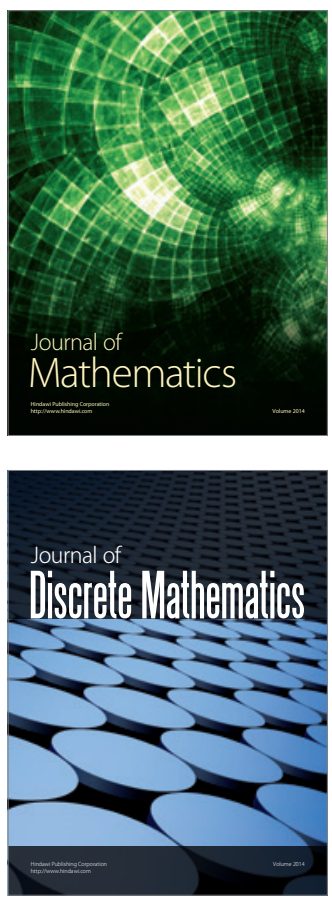

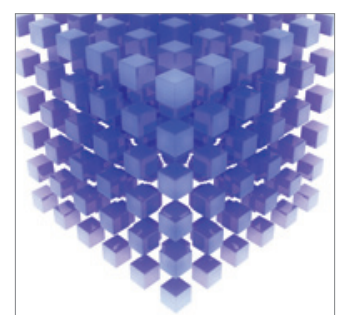

Mathematical Problems in Engineering
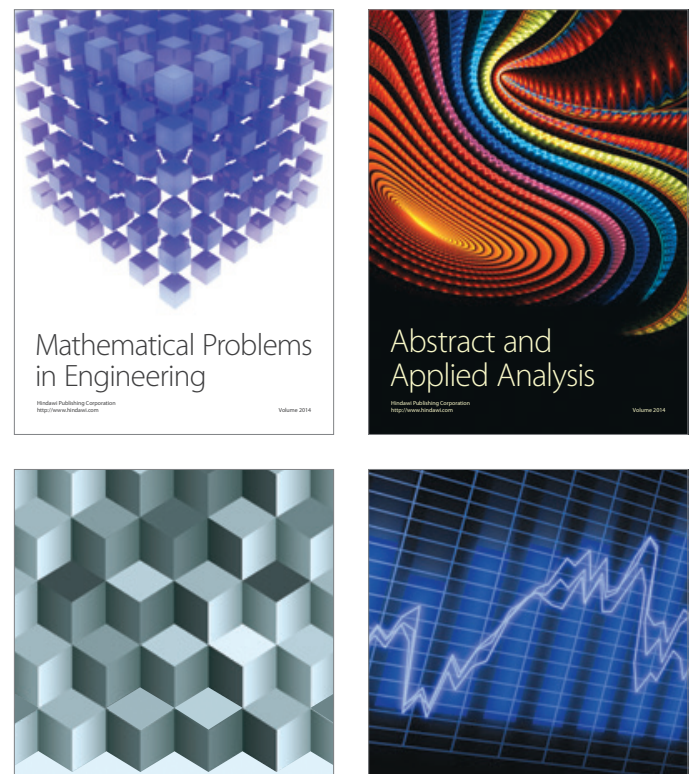

Journal of

Function Spaces

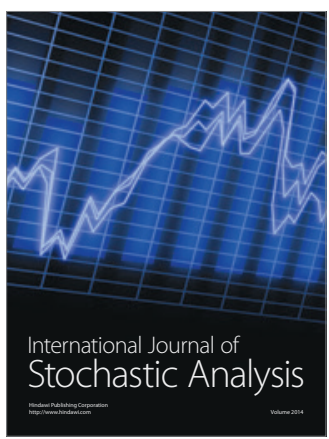

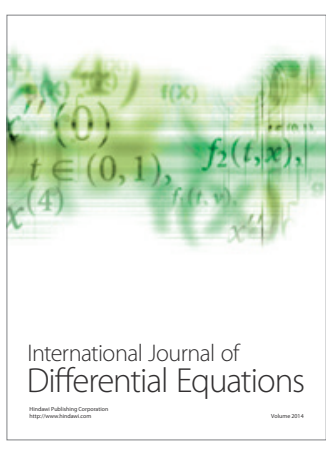
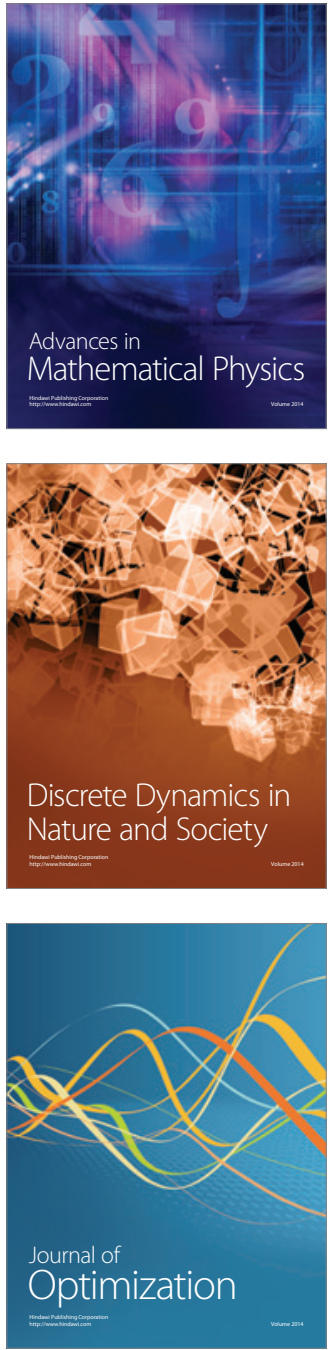